\title{
Experimental Setup with Chaotic and Periodic Excitations for Cell Growth Studies
}

\author{
Victor P. Cojocaru ${ }^{1,2}$ \\ ${ }^{1}$ D. Ghitu Inst. Electronic Engineering and \\ Nanotechnologies, ${ }^{2}$ Technical University of Moldova \\ Chisinau, Moldova \\ vcojocaru@nano.asm.md
}

Jurijs Dehtjars

Institute of Biomedical Engineering and Nanotechnologies, Riga Technical University Riga, Latvia jurijs.dehtjars@rtu.lv

\author{
Horia-Nicolai L. Teodorescu ${ }^{3,4}$ \\ ${ }^{3}$ Gheorghe Asachi Technical University of Iasi \\ ${ }^{4}$ Inst. of Computer Science of the Romanian Academy \\ Iasi, Romania \\ Alexander Rapoport \\ Institute of Microbiology and Biotechnology, \\ University of Latvia \\ Riga, Latvia \\ rapoport@latnet.lv \\ Annamarija Starsaja \\ Institute of Biomedical Engineering and \\ Nanotechnologies, Riga Technical University \\ Riga, Latvia \\ annamarija.starsaja@gmail.com
}

\begin{abstract}
The paper presents circuits used for excitation living cells to increase their growth rate. The main novelty is the proposal of using chaotic oscillations for the electromagnetic excitation. The research is in a preliminary phase and no conclusions have been yet derived for applications in biotechnology.
\end{abstract}

Keywords- chaotic generators; harmonic oscillators; cell growth stimulation; biotechnology

\section{INTRODUCTION}

Electromagnetic radiation is used to help grow cells for biotechnologies [1-8]. Various frequencies and waveforms have been tested; for example [1] reports on the effects of Millimeter waves or extremely high frequency electromagnetic fields; [2] reviews the use of electric, magnetic, and electromagnetic field for directed cell migration in medicine, [3] reports on the effects of low-level 900 $\mathrm{MHz}$ electromagnetic fields on vegetal tissue, [6] reports on bactericide effects, [7] presents osteogenesis effects, while [4] studies the response of yeast saccharomyces cerevisiae. However, the effect of chaotic electromagnetic radiation (CEMR) on the cells was not studied. The present article aims to propose, design, and demonstrate two types of CEMR generators and show their application in promoting the growth of colonies of yeast cells.

\section{CIRCUITS AND CIRCUIT ANALYSIS}

\section{A. Circuits for Periodic Excitation-Colpitts oscillator in periodic regime}

We have chosen a Colpitts oscillator circuit with a common base since it easily achieves high-frequency oscillations. No buffer is used as an interaction of the load with the oscillator was desired in this experimental phase, but a buffered version is considered for comparison. Hartley oscillators could have been also used in the application, but we preferred Colpitts oscillators because it is easier to tune them by changing a capacitor instead of the tapping point of an inductance. Also, Colpitts oscillators are said to be more stable at high frequencies; a stable operation, especially in the chaotic regime where the operation is very sensitive, is necessary for the reproducibility of the results.

In the Colpitts oscillator, a cascade with a common base does not shift the phase. The $\mathrm{L}_{1} \mathrm{C}_{1} \mathrm{C}_{2}$ circuit is fully connected to the collector. The emitter is connected to the circuit to the midpoint of the capacitive voltage divider with phase imbalance; with equal $\mathrm{C}_{1}$ and $\mathrm{C}_{2}$, the phase imbalance and the loop phase shift is $45^{\circ}$. The oscillating frequency, as well known, is $\omega_{0}=1 / \sqrt{L \times\left(C_{1}, C_{2}\right)}$, where $\left(C_{1}, C_{2}\right)$ denotes the series circuit. The circuit can achieve (almost perfectly) sinusoidal oscillations as long as the nonlinear interval of operation of the transistor is not reached. The operation of the circuit is largely determined by the $\mathrm{Q}$ factor of the tank circuit (L, $\left.\left(C_{1}, C_{2}\right)\right)$, [8], [9]. Notice that any load at the output of the circuit modifies the operation. A purely capacitive load placed as in Fig. 1 will create a short-circuit condition at higher frequencies. We are interested in this paper especially in the chaotic regime, as discussed subsequently.

\section{B. Circuits for Excitation with Chaotic Signals}

Two circuits are used for chaos generation, the Colpitts oscillator and a circuit proposed in [9]. The same Colpitts oscillator can be used to generate chaos, because the circuit has a nonlinear element (the transistor), $I_{E} \approx I_{S} \exp \left(\frac{V_{B E}}{V_{T}}\right)$ for $V_{B E} \gg V_{T}$, and is described by a third-order equation [8]. The equation for this configuration is [8]:

$$
f\left(V_{C_{2}}\right)=I_{S} \exp \left(-\frac{V_{C_{2}}}{V_{T}}\right)
$$




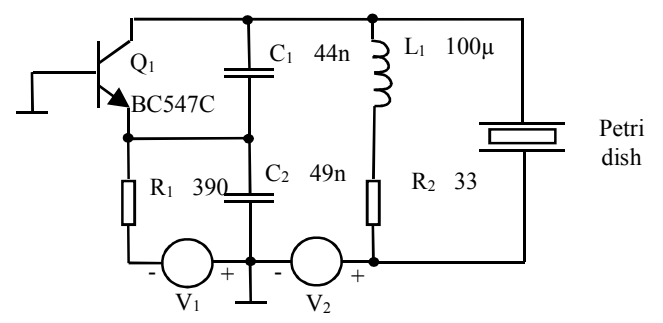

Figure 1. The Colpitts oscillator circuit and its connection to the capacitance including the Petri dish for cell growth

In Fig. 2, $L$ and $R$ are the parasitic inductance and the resistance of the measurement cell and of the cables. To minimize these parasitic elements, the cable from the Colpitts oscillator to the measuring capacitor should be as short as possible. The oscillator should actually be placed on the active plate of the capacitor.

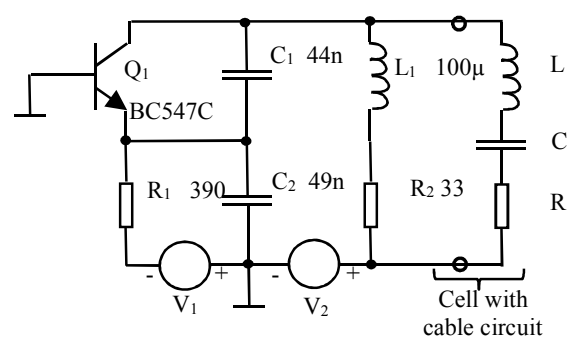

Figure 2. Oscillator with the equivalent circuit of the load (basic approximation of the load).

It is well known that Colpitts oscillator has a rich behavior and bifurcation diagram, with multiple intervals of periodic and chaotic regimes [8]. The state equations derived by [8] describe well the common base configuration. These equations are reproduced below (from [8]):

$$
\begin{gathered}
\frac{C_{1} d V_{C_{1}}}{d t}=-f\left(V_{C_{2}}\right)+I_{L}, C_{2} \frac{d V_{C_{2}}}{d t}=I_{L}-I_{0} \\
L \frac{d I_{L}}{d t}=-V_{C_{1}}-V_{C_{2}}-R I_{L}+V_{c c}
\end{gathered}
$$

where, for adapting to the circuits in Fig. 1 and Fig. 2, $I_{0}=V_{1} / R_{1}$, assumed a constant current, and $V_{c c}=V_{2}$. However, these equations describe the idealized case of no load to the oscillator. When a capacitive load, $C_{l}$, is added, as in Fig. 2, but without parasitic elements $(\mathrm{R}, \mathrm{L})$, the third equations above have to be replaced by

$$
\begin{gathered}
\frac{C_{1} d V_{C_{1}}}{d t}=-f\left(V_{C_{2}}\right)+\left(I_{L}+I_{C}\right) \\
C_{2} \frac{d V_{C_{2}}}{d t}=I_{L}+I_{C}-I_{0} \\
L \frac{d I_{L}}{d t}=-V_{C_{1}}-V_{C_{2}}-R I_{L}+V_{c c} \\
\frac{C d V_{C}}{d t}=I_{C}, V_{c}=L \frac{d I_{L}}{d t}+R I_{L}
\end{gathered}
$$

The addition of the capacitive load increases the order of the equivalent equation of the Colpitts circuit by 1 , making the dynamics more intricate. Adding the parasitic elements of the capacitive probe (with Petri dish) further complicates the analysis; therefore, we will assume these elements null.

In essence, Colpitts oscillators can behave as a multi-pulse periodic oscillator, where the multi-pulse number depends on the operation in the cut-off region of the transistor [8], [9]. For an example, see the next Section (Fig. 4, left). In essence, this operation corresponds to periodical bursts. Maggio et al [8] suggests that there may be an infinite family of multipulse periodic solutions. This may imply that the family is countable, because two solutions differ only by the number of counts, but we argue that in fact the family may be uncountable, with solutions differing continuously by their waveforms.

Further design issues on chaotic circuits are given for example in [11-14]. It would be interesting in the future to test the Colpitts variant proposed in [14].

\section{The Second Circuit Used}

The second circuit is a copy of one we previously applied in another application [10]; the circuit is shown in Fig. 3.

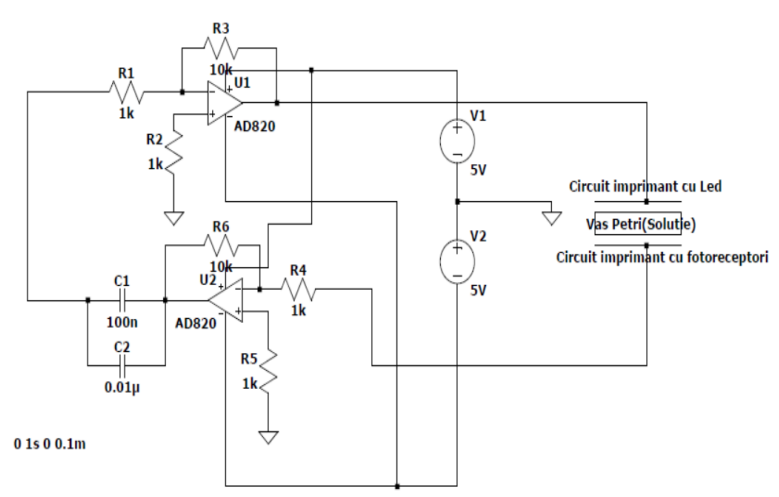

Figure 3. The second chaotic signal generator used in tests. See [10] for details.

The chaotic circuit largely reproduces the one we conceived for a bio-mimetic sensor for water salinity [10]. It consists of two operational amplifiers coupled in a loop through two circuits based on capacitors. The first coupling circuit consists in one electrolytic and one non-electrolytic capacitor coupled in parallel, and the resulted equivalent capacitor is in series with a resistor. The second coupling circuit is the probe, which consists of the excitation capacitance and the Petri dish with its content. The two capacitors in parallel have an intricate equivalent scheme, including nonlinear capacitances and resistances and linear inductances. Their role is critical in the generation of chaos. The two operational amplifiers have high input impedances.

\section{CIRCUITS OPERATION AND TESTING}

In this section, a few examples of operation are given. An example of a bi-pulse operation is shown in Fig. 4 (left).

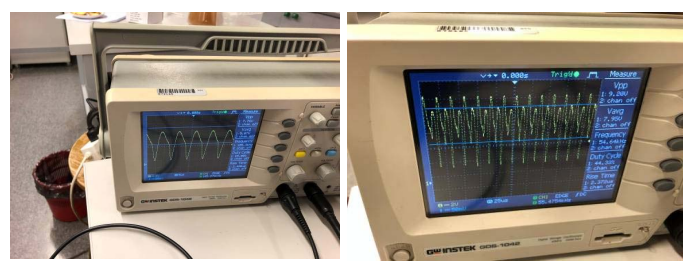

Figure 4. Example of a waveform used in tests. Left: The waveform corresponds to a period one oscillation, see [8]. Right The waveform corresponds to a period two oscillation (see [8]). 
The simulations show that the capacitive load changes the dynamics of the oscillator, as predicted by the equations (Fig. 5). However we believe that the simulations do not grasp the entire process, because of idealized schemes for the components, without parasitic elements.
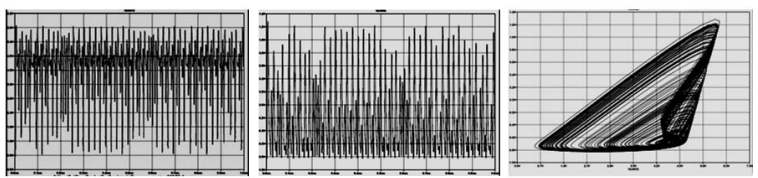

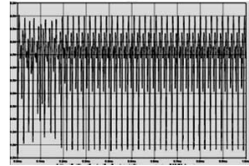

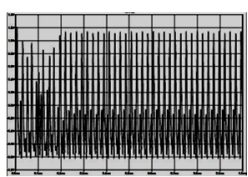

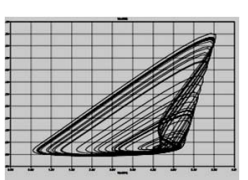

Figure 5. Simulation results. Upper panel: no load. Lower panel: with 100 pF load. (a) Collector-GND, (b) Emitter-GND, (c) Phase portrait.

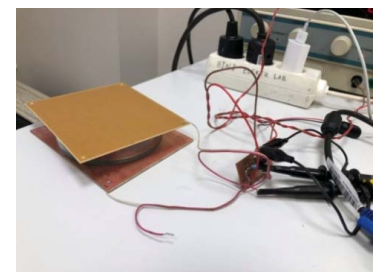

Figure 6. View of the excitation capacitor (on PCB) with the Petri dish inside.

\section{CELl EXCITATION AND MONITORING}

Yeast cells Saccharomyces cerevisiae 14, which were obtained from the Latvian Microorganism Culture Collections of the University of Latvia, are used for the experiment. Yeast Peptone Glucose (YPG) agar, with the composition given in Table 1, is the recommended nutrient medium for the cultivation of Saccharomyces cerevisiae yeast cells for molecular biology. The yeast extract allows the cells to grow faster so that the cells divide every 90 minutes during exponential or log phase growth. Peptone, the peptic digestion of animal tissues, is a source of carbon, nitrogen, vitamins and minerals, while glucose is a source of cellular energy [15].

TABLE I. COMposition In The Petri Dish

\begin{tabular}{|l|l|}
\hline Component & Amount (g) for $\mathbf{1 0 0 0} \mathbf{~ m l}$ \\
\hline Yeast extract & 10 \\
\hline Peptone & 20 \\
\hline Glucose & 20 \\
\hline Agar & 20 \\
\hline
\end{tabular}

For the convenience of the experiment, several 50 $\mathrm{ml}$ boiling flasks of agar were formed, the composition ratio in this case is: $0.5 \mathrm{~g}$ yeast extract, $1 \mathrm{~g}$ peptone, 1 $\mathrm{g}$ glucose, $1 \mathrm{~g}$ agar. All the ingredients were weighed on an electronic scale and placed in several flasks; using a measuring cylinder, $50 \mathrm{ml}$ of distilled water was added to them. After that the resulting emulsion was mixed thoroughly. The resulting solution was sterilized in an autoclave under elevated temperature and pressure.

Hot agar was poured into a $60 \mathrm{~mm}$ diameter, 15 $\mathrm{mm}$ high Petri dish in which cells were seeded after agar solidification. Agar flask was closed tightly with a cotton-wool stopper to maintain its sterility. The cells were seeded as follows: in a laminar flow cabinet, under sterile conditions, right next to the flame of an alcohol lamp, using a sterile inoculation loop, a colony was taken from the existing cell culture and brought to a new petri dish with agar. Subsequently, paraffin was put around the closed petri dish to maintain sterile conditions. The newly formed cell culture was placed in an incubator at $30^{\circ} \mathrm{C}$ for 48 hours, with agared side down and lid up to prevent water condensate from accumulating on the agar and cells. After the cells were growing in incubator for 48 hours, the Petri dish is then transferred to a refrigerator, where the cell culture can be stored for one month without transfer.

The cell suspension was prepared using the previously established cell culture and saline $(0.9 \%$ sodium chloride in water). Using a pipette, $1 \mathrm{ml}$ of saline was added to a $1.5 \mathrm{ml}$ plastic centrifuge tube in a laminar flow cabinet near the alcohol lamp. Next, in previously made Petri dish was lightly touched with a sterile inoculation loop, so that some cells remain on it, then the loop was soaked in the saline of the tube and mixing well with the liquid. It is important to mix the cell pellet in solution using the LU DAC Bio Vortex V1 mixing device.

Before forming the sample, it is necessary to melt the agar. The agar flask was placed into the microwave and set the timer for 2 minutes. Every 10 seconds, the microwave was opened and the flask was shook to mix the agar and to prevent the stopper from flying out of the flask, spilling out the agar. Heating of the agar is stopped when it is completely liquid or is already bubbling. After brief cooling, it is poured evenly onto previously cleaned glass slides in a laminar flow cabinet (Fig. 6). Once the agar has solidified on the slide, the area where the agar is most uniform was selected and a square of the uniform agar was cut with a scalpel and excess agar was removed. Then, two rectangles were cut on the sides of the agar square to provide air to the yeast cells in the sample.

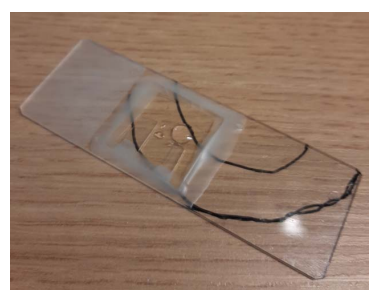

Figure 7. Glass plate with cells as used in experiments. The line draw indicate the approximate position of the capacitor plates.

Finally, using an inoculation loop from the suspension tube the suspension was placed in several places on the agar square, between the cut-out rectangles. The square was then covered with a $0.22 \times 0.22 \mathrm{~mm}, 0.17 \mathrm{~mm}$ thin coverslip and liquid absorbent paper was used to remove excess liquid along the edges of the coverslip and slightly inside the agar. This prevented the cells from floating on the agarized surface - they must be fixed. To maintain all the necessary conditions for the cells, melted paraffin was smeared around the edges of the coverslip. Paraffin prevents external conditions to negatively 
affect the cell environment and its growth; it also keeps the coverslip stable in place on the large slide.

To observe the cells, the optical microscope OLYMPUS BX51 of the LU DAC microbiology laboratory was used. It is also equipped with a video camera. Before placing the sample on an optical microscope table, immerse Karl ROTH Art. X8991 was put on the sample coverslip, which is required for the UPlanFLN lens to slide easily over the sample surface at $\times 100$ magnification. For taking pictures with the microscope, the computer program "cell $\wedge$ F" was used.

The $(x, y)$ coordinates of the location of each cell were recorded from the microscope table. Pictures were taken with different focuses to make it as easy as possible to find the cells in the next measurement.

\section{EXAMPLES OF PRELIMINARY TESTS ON CELLS}

While it is too early to derive conclusions, because all results are incipient, we illustrate the principle of the experiments in the preliminary form, with pictures (Figs. 8,9, and 10).

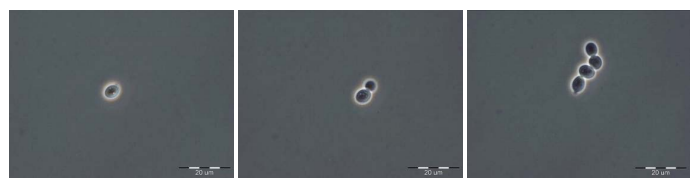

Figure 8. (a) Initial cell (b) Cell division after irradiation with chaotic signal for $60 \mathrm{~min}$. (c). Cell division after heating in the thermostat at $30^{\circ} \mathrm{C}$ for $120 \mathrm{~min}$.
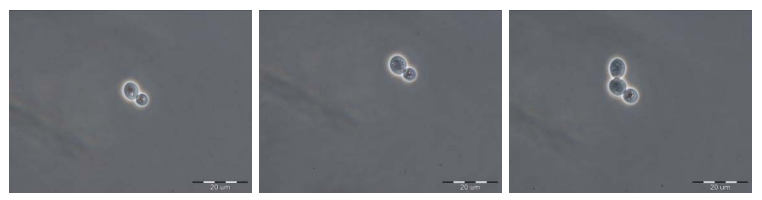

Figure 9. (a) Initial cell (b) Cell division after irradiation with periodic signal for $60 \mathrm{~min}$. (c). Cell division after heating in the thermostat at $30{ }^{\circ} \mathrm{C}$ for $120 \mathrm{~min}$.
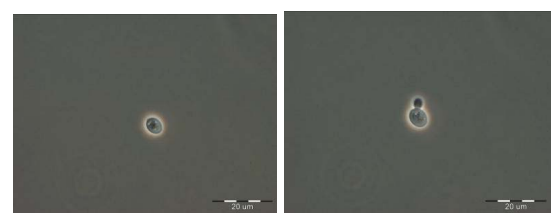

Figure 10. (a) Initial cell (b) Cell division after heating in the thermostat at $30{ }^{\circ} \mathrm{C}$ for $120 \mathrm{~min}$.

The preliminary experiments show that there are differences between cells growth under the different condition (excitations).

\section{CONCLUSIONS}

In summary, we have proposed a new method, based on chaotic signals, for promoting the cell growth for biotechnology applications and suggested two suitable chaotic circuits for this purpose. The design was accompanied by simulations. The experiments confirmed the circuits' designs. Preliminary results with cell growth in a demonstrator were also presented. Further studies are needed to improve the chaos generators and to produce reliable experimental results on the cell growth with the presented method and circuits. A detailed comparison of the results obtained with the two circuits is also needed.

\section{ACKNOWLEDGMENT}

V.C. was supported by the grant VIAA, State Education Development Agency of Latvia internship.

Authors' contributions. V Cojocaru, J. Dehtjars and A. Rapaport proposed the topic and contributed Sections IV and V. A. Starsaja helped with the experiments on cells. HN Teodorescu wrote the Section I and II and contributed to Sections III and VI. He takes no responsibility for the other sections. V Cojocaru built all circuits and performed the experiments with the help of the Latvian authors.

\section{REFERENCES}

[1] Soghomonyan, D., Trchounian, K. \& Trchounian, A. "Millimeter waves or extremely high frequency electromagnetic fields in the environment: what are their effects on bacteria?" Appl Microbiol Biotechnol 100, 47614771 (2016). https://doi.org/10.1007/s00253-016-7538-0.

[2] Ross, C.L. (2017), "The use of electric, magnetic, and electromagnetic field for directed cell migration and adhesion in regenerative medicine." Biotechnol Progress, Vol 33, Issue 1, pp. 5-16. doi:10.1002/btpr.2371.

[3] M. Răcuciu, S. Miclăuş, "Low-Level 900 Mhz Electromagnetic Field Influence on Vegetal Tissue," Rom. J. Biophys., Vol. 17, No. 3, 149-156, 2007.

[4] Hunt, R.W.; Zavalin, A.; Bhatnagar, A.; Chinnasamy, S.; Das, K.C. Electromagnetic biostimulation of living cultures for biotechnology, biofuel and bioenergy applications. Int. J. Mol. Sci. 2009, 10, 4515-4558.

[5] M. Mehedintu, H. Berg, Proliferation response of yeast saccharomyces cerevisiae on electromagnetic field parameters, Bioelectrochemistry and Bioenergetics, Vol 43, Issue 1, 1997, pp. 67-70, ISSN 0302-4598.

[6] H. Torgomyan \& A. Trchounian (2013) Bactericidal effects of low-intensity extremely high frequency electromagnetic field: an overview with phenomenon, mechanisms, targets and consequences, Critical Reviews in Microbiology, 39:1, 102-111, DOI: 10.3109/1040841X.2012.691461.

[7] Arjmand, M, Ardeshirylajimi, A, Maghsoudi, H, Azadian, E. Osteogenic differentiation potential of mesenchymal stem cells cultured on nanofibrous scaffold improved in the presence of pulsed electromagnetic field. J Cell Physiol. 2018; vol. 233, 1061-1070. https://doi.org/10.1002/jcp.25962.

[8] Maggio, G.M., De Feo, O., Kennedy, M.P., 1999. Nonlinear analysis of the Colpitts oscillator and applications to design, IEEE Transactions on Circuits and Systems I: Fundamental Theory and Applications, 46 (9), pp. 1118-1130, Sep 1999.

[9] M. P. Kennedy, Chaos in the Colpitts Oscillator. IEEE Trans. Circuits Systems-I: Fundamental Theory \& Applications, Vol. 41, No. 11, Nov 1994, pp. 771-774.

[10] H.-N.L. Teodorescu, V.P Cojocaru, Biomimetic chaotic sensors for water salinity measurements and conductive titrimetry. 2012 Third International Conference on Emerging Security Technologies, 2012/9/5, 182-185.

[11] Z. G. Shi \& L. X. Ran (2006) Microwave chaotic Colpitts oscillator: Design, implementation and applications, $J$. Electromagnetic Waves and Applications, 20:10, 1335-1349, DOI: 10.1163/156939306779276802.

[12] Andreani, P., Wang, X., Vandi, L., \& Fard, A. (2005). A study of phase noise in Colpitts and LC-tank CMOS oscillators. IEEE J. Solid State Circuits, 40(5), 1107-1118. https://doi.org/10.1109/JSSC.2005.845991

[13] G.H. Li, S.P. Zhou, K. Yang, Controlling chaos in Colpitts oscillator. Chaos, Solitons \&Fractals, vol. 33, 2007, 582-587.

[14] A. Kartci, U. Engin Ayten, N. Herencsar, R. Sotner, J. Jerabek, K. Vrba. Floating capacitance multiplier simulator for grounded RC Colpitts oscillator design. Proc. AE 2015.

[15] https://legacy.bd.com/europe/regulatory/Assets/IFU/Difco_B BL/242820.pdf. 